\title{
Brain imaging in pediatric cancer survivors: correlates of cognitive impairment
}

\author{
Shelli R. Kesler ${ }^{1}$, Charlotte Sleurs ${ }^{2,3}$, Brenna C. McDonald ${ }^{4}$, Sabine Deprez ${ }^{3,5}$, \\ Ellen van der Plas $^{6}$, Brian J. Nieman ${ }^{7}$
}

1. University of Texas at Austin, School of Nursing, Department of Diagnostic Medicine, Dell School of Medicine, LIVESTRONG Cancer Institutes (srkesler@austin.utexas.edu, ORCID 0000-0002-4745-8014)

2. Catholic University of Leuven, Department of Oncology (charlotte.sleurs@kuleuven.be, ORCID 0000-0002-4480-8330)

3. Leuven Cancer Institute, Leuven, Belgium

4. Indiana University School of Medicine, Department of Radiology and Imaging Sciences, Center for Neuroimaging, Indiana University Melvin and Bren Simon Cancer Center (mcdonalb@iupui.edu, ORCID 0000-0001-6895-4490)

5. Catholic University of Leuven, Department of Imaging \& Pathology (sabine.deprez@kuleuven.be, ORCID 0000-0001-7451-5355)

6. University of lowa Hospital \& Clinics, Department of Psychiatry (ellen-vanderplas@uiowa.edu; ORCID: 0000-0002-7490-6636)

7. Hospital for Sick Children, Translational Medicine; University of Toronto, Department of Medical Biophysics; Ontario Institute for Cancer Research

Revised Submission to Journal of Clinical Oncology (September 2020) Special Issue on Childhood Cancer Survivorship

\section{Funding Acknowledgements:}

SRK acknowledges support from National Institutes of Health: National Cancer Institute grants R01CA226080 and R01CA172145. CS acknowledges support from the Kinderkankerfonds Leuven. BCM acknowledges support from the National Institutes of Health: National Cancer Institute grants R35CA197289, R01CA129769, and R01CA244673 and National Institute on Aging grants P30AG010133 and R01AG019771. BJN acknowledges support from the Ontario Institute for Cancer Research through funding provided by the Government of Ontario (IA-024) and by the Canadian Institutes of Health Research (156250, 158622, 168925).

\section{Corresponding Author:}

Shelli R. Kesler, PhD

Department of Diagnostic Medicine, Dell School of Medicine

The University of Texas at Austin

1710 Red River St, RM 3.426, Austin TX 78712

512-232-5292

srkesler@austin.utexas.edu 


\section{Introduction}

Pediatric cancer and its treatment are associated with increased risk for acute and chronic cognitive impairments. Neuroimaging studies indicate a common biologic pathway of candidate mechanisms underlying these impairments is diffuse brain injury (see Sleurs et al. for review $\left.^{1}\right)$. Brain injury is frequently assessed using neuroimaging biomarkers which improve understanding of neurologic and psychiatric pathologies beyond current diagnostic boundaries, ${ }^{2}$ provide unique insights regarding cognitive changes, and specify therapeutic targets for these changes. ${ }^{3}$ Neuroimaging metrics can also serve as biologic outcome measures for interventions by quantifying treatment impacts on previously identified neural dysfunctions, which may guide treatment refinement. ${ }^{3}$ Moreover, neuroimaging outcomes may be superior to behavioral and other metrics for predicting future cognitive outcomes. ${ }^{4}$

As a result of this potential, neuroimaging has an expanding role in study and treatment of long-term cognitive impairments in childhood cancer survivors. Radiological observation by magnetic resonance imaging (MRI) in this population sometimes reveals leukoencephalopathy, ${ }^{5}$ which has been associated with risk for subsequent behavioral problems. ${ }^{6}$ Childhood cancer survivors are also at elevated risk for cerebrovascular disease, ${ }^{7}$ and can exhibit impaired cerebral blood flow. ${ }^{8}$ Advanced, quantitative comparisons reveal differences in otherwise normal-appearing brain tissue, which are the focus of this paper. We review recent clinical and preclinical neuroimaging studies of these brain structure and function alterations and discuss the insights they provide regarding cognitive impairment. Table 1 provides a descriptive summary of the imaging modalities and outcome measures to be discussed. Figure 1 highlights key neuroimaging findings.

\section{Structural Alterations in Gray and White Matter}

Anatomical MRI scans from childhood cancer survivor populations can be processed to extract quantitative measurements of brain geometry for comparison to control groups for identification of neurodevelopmental alterations (see Figure 2). As would be expected, brain tumors and their treatment lead to the most prominent alterations in neurodevelopment. Tumor-related complications such as elevated intracranial pressure, residual lesions, and 
hydrocephalus can all lead to major structural deformations affecting the brain tissue and cerebrospinal fluid spaces. In addition to the effects from the tumor itself, neurosurgery-, radiation- and/or chemotherapy-induced neural injury can impair neurodevelopment and affect long-term intellectual ability. Most neuroimaging studies of pediatric brain tumor survivors have focused on medulloblastoma, the most common primary CNS malignancy in childhood. The infratentorial tumor location of these tumors facilitates investigation of supratentorial brain changes. Mulhern et al. (2001) demonstrated that medulloblastoma patients, who are routinely treated with chemotherapy and craniospinal irradiation (CSI), are at highest risk for cognitive decline if diagnosed and cranially irradiated at younger ages. ${ }^{9}$ Smaller white matter (WM) volume largely accounted for these effects, and is also linked with impaired attention. ${ }^{10}$ In addition to WM volumetric changes, some studies also evidenced hippocampal volume loss ${ }^{11}$ and thinner ${ }^{12}$ or thicker ${ }^{13}$ cortices shortly after treatment of medulloblastoma, as compared to healthy children. Given that CSI dose plays an important role in cognitive and intellectual outcomes of brain tumor patients, CSI is limited in younger children when possible. CSI was also associated with brain structure and cognitive function differences in acute lymphoblastic leukemia (ALL) survivors, ${ }^{14}$ and has almost entirely been replaced by CNS-directed chemotherapy in current treatment regimens for this population.

Even with chemotherapy-only ALL treatment protocols, brain structure alterations are evident using quantitative or voxel-based comparisons, ${ }^{15}$ albeit to a lesser extent. ${ }^{16}$ Most studies showed smaller WM volume, ${ }^{16-20}$ but cortical geometry has received increasing attention, with smaller gray matter volumes ${ }^{17,18}$ and differences in cortical thickness ${ }^{21,22}$ being reported. Interestingly, a recent study by Philips et al. (2020) explored the impact of systemic glucocorticoid treatment with dexamethasone on structural development in childhood ALL survivors, ${ }^{23}$ identifying an association between high serum concentration and thinner entorhinal and frontal cortices in females. Much less attention has been paid to potential neurotoxic processes resulting from chemotherapy in non-CNS solid tumor patients. Nevertheless, long-term cortical thinning in frontal, cerebellar, and parahippocampal areas was recently demonstrated in sarcoma patients. ${ }^{24}$ Even though such systemic treatment effects often appear to be diffuse in non-CNS childhood cancer patients, ${ }^{12,18}$ hippocampal and frontal 
regions may be particularly vulnerable. Cognitive changes associated with these structural brain alterations are more commonly reported in domains of memory, ${ }^{14}$ processing speed ${ }^{16,17}$ and executive function. ${ }^{18,21}$

\section{Microstructural White Matter Change}

Damage to WM bundles can decrease the efficiency of communication between neural systems and therefore impair cognitive functioning. While volumetric investigations allow quantification of macrostructural WM injury, they do not provide detailed microstructural information indicative of WM health. Diffusion-weighted imaging techniques enable characterization of WM architecture based on the diffusion of water molecules, which is affected by the morphology of axonal membranes and myelin sheaths. Modeling based on acquired diffusion MR images provides estimates of a number of metrics indicative of WM health (see Figure 3).

Using diffusion tensor imaging (DTI, the simplest diffusion model in widespread use), lower fractional anisotropy (FA), higher mean diffusivity (MD), and higher radial diffusivity (RD) are often detected in the WM of survivors who received cranial irradiation for treatment of pediatric brain tumors or ALL. Microstructural differences are mainly found in frontal, parietal, and temporal WM or their connecting bundles, ${ }^{25-28}$ from a few years ${ }^{27,28}$ to more than 25 years post-treatment. ${ }^{25,26}$ Younger age at $\mathrm{CSI}^{25,29}$ and higher radiation dose $25,29,30$ were associated with more WM microstructural changes. Additionally, several studies reported the corpus callosum to be particularly vulnerable to radiation injury. ${ }^{27,30,31}$ Several studies evaluating the impact of CNS-directed therapy without CSI also report significantly lower FA and higher mean and/or radial diffusivity, both in $\mathrm{ALL}^{6,32-35}$ and in low-grade glioma survivors. ${ }^{36,37}$ The amount of WM change depends on the administered chemotherapy protocol, ${ }^{34}$ exposure to intrathecal methotrexate, ${ }^{22,33,35}$ and associated anesthesia. ${ }^{38}$

WM microstructural changes after CSI and chemotherapy in survivors of pediatric brain tumors and ALL have frequently been associated with decreased memory function, 26,37 processing speed, ${ }^{28,32,34,35,37}$ attention, ${ }^{25}$ executive functioning, ${ }^{39,40}$ and IQ. ${ }^{27,}{ }^{29}$ Lower frontal WM FA in pediatric medulloblastoma patients following surgery but before irradiation or 
chemotherapy was associated with decreased executive functioning 36 months later, ${ }^{41}$ suggesting an important impact of the tumor and/or its surgical removal on cognitive development. Additionally, lower FA in fronto-occipital fasciculus before/immediately after radiation in posterior fossa tumors predicted decline in processing speed and executive functioning over time. ${ }^{42}$

While DTI has the sensitivity to detect microstructural WM damage, it does not distinguish the type of damage that occurred (e.g., myelin damage vs. axonal injury). Higherorder diffusion models can provide such additional insights. Follin et al. ${ }^{26}$ reported a decrease in mean kurtosis 34 years after CSI, which could indicate tissue simplification with neuronal shrinkage and decreased axonal density. Billiet et al. ${ }^{39}$ observed increased FA and lower orientation dispersity index (ODI) in the centrum semiovale of ALL survivors, which was attributed to the effects of several crossing fiber bundles and possible axonal alterations after intrathecal methotrexate without CSI. In another study, Sleurs et al. ${ }^{43}$ combined different advanced diffusion models to investigate WM microstructural changes after systemic intravenous chemotherapy in bone and soft tissue sarcoma survivors. They reported extensive regions showing lower FA overlapping with changes in parameters derived from neurite orientation dispersion and density imaging and from fiber orientation-based analyses. Additionally, lower apparent fiber density in the corpus callosum was significantly predicted by chemotherapy treatment and correlated with time since diagnosis and outcomes of neurocognitive tests. More recently, the organization of the WM connectome has been investigated, by applying graph theory analysis to diffusion measures. Altered WM topology was found in adult survivors of pediatric brain tumors ${ }^{44}$ and $A L L,{ }^{45}$ which may suggest network reorganization and underlie cognitive impairment.

To further disentangle the underlying neural correlates of the observed WM microstructural changes, techniques that are sensitive to changes in myelin, such as myelin water imaging and magnetization transfer imaging, are promising. So far, only a few conflicting results have been reported. While two studies did not detect differences in myelin water ${ }^{39}$ or magnetization transfer ${ }^{18}$ measures between ALL survivors receiving methotrexate and controls, 
Yamamoto et al. ${ }^{46}$ did find decreased peak values in magnetization transfer ratio histograms post-methotrexate, suggestive of demyelination.

\section{Functional Changes}

Functional neuroimaging provides insight into neural correlates of cognitive changes related to pediatric cancer and its treatments. For example, fluorodeoxyglucose positron emission tomography (PET) has demonstrated altered brain metabolism after chemotherapy in children with Hodgkin and non-Hodgkin lymphoma. ${ }^{47,} 48$ Magnetoencephalography (MEG) showed a trend toward global slowing of brain oscillatory activity in adult survivors of ALL treated with CSI and chemotherapy relative to controls, accompanied by significantly poorer performance on measures of cognitive flexibility, visuomotor accuracy, and visuospatial working memory. ${ }^{49} \mathrm{ALL}$ survivors treated with chemotherapy only did not show significant differences. To date, however, studies employing fMRI have been much more prevalent.

Task-based fMRI detects brain activation associated with performance of a particular activity. Pediatric brain tumor survivors (mixed pathologies, treated with resection with/without chemotherapy/CSI) showed lower bilateral frontal activation and increased left cingulate activation during working memory processing relative to controls. ${ }^{50}$ Better cognitive performance correlated with higher frontal and lower cingulate activation. In contrast, adult survivors of posterior fossa tumors (treated with resection with/without chemotherapy/CSI) showed greater left frontal and parietal working memory-related activation relative to controls, which correlated with poorer working memory performance. ${ }^{51}$ Greater working memoryrelated $\mathrm{fMRI}$ activation was associated with better working memory performance and motor proficiency in children newly diagnosed with craniopharyngioma (pre-systemic treatment), who also exhibited significantly poorer aerobic fitness, motor proficiency and working memory. ${ }^{52}$ In large cohorts of ALL survivors treated with chemotherapy only, activation during attentional tasks was associated with age at diagnosis and methotrexate exposure. ${ }^{22,53}$

Resting-state fMRI (rsfMRI) measures correlated neural activity in the absence of a task. In children newly diagnosed with ALL (pre-systemic treatment), rsfMRI showed regions of decreased or increased regional homogeneity relative to controls, in the absence of differences 
in intellectual ability. ${ }^{54}$ In adult survivors of childhood cerebellar tumors (mixed pathologies, treated with resection with or without chemotherapy/CSI), rSfMRI showed hyperconnectivity in multiple brain networks relative to controls. ${ }^{55}$ In a large sample of childhood ALL survivors treated with chemotherapy only, those with executive dysfunction showed lower global efficiency of structural and functional connectomes than those without executive dysfunction, as well as hyperconnectivity of various mesial and lateral temporal brain regions and poor separation of brain networks. ${ }^{56}$ Lowest network efficiencies were found in patients with highversus low-risk disease and those receiving more intrathecal methotrexate treatments. Adult ALL survivors treated with chemotherapy only showed lower functional connectivity between the default mode network and inferior temporal gyrus relative to controls, along with alterations in WM that correlated with a measure of cognitive flexibility. ${ }^{39}$

Both $\mathrm{FMRI}$ and MEG have been used in evaluating the impact of rehabilitative treatments. In pediatric medulloblastoma survivors who received a reading intervention during radiation (after resection/before chemotherapy), task-based fMRI 2-3 years post-treatment suggested normalization of activation in patients who received the reading intervention relative to those who did not. ${ }^{57}$ Children treated for brain tumors (mixed pathologies) with surgery/CSI with/without chemotherapy showed altered MEG functional connectivity pre- to post- aerobic exercise intervention, suggesting enhanced controlled attention/response inhibition. ${ }^{58}$ In a pediatric cancer population with mixed pathologies and treatments, continuous performance task fMRI activation was lower relative to controls prior to cognitive remediation, with increases post-treatment. ${ }^{59}$ In contrast, in a similarly mixed sample, those who received computerized cognitive training showed lower frontal working memory-related activation preto post-treatment, along with improved working memory performance and parent-rated executive function. ${ }^{60}$ Activation correlated with attentional performance.

\section{Imaging as a Translational Tool: Animal Models}

An advantage of neuroimaging tools is that they can also be applied to animal models of childhood cancer and its treatment. Though differences between species are important to recognize, animal models provide opportunities to link imaging findings to underlying 
pathology, to evaluate mechanisms of brain toxicity, and to assay candidate treatments. A mouse model of pediatric cranial radiotherapy (CRT), for instance, shows similar structural changes to those observed in humans, ${ }^{13}$ including smaller white and gray matter volumes that depend on age at treatment, dose, and sex. ${ }^{61,62}$ The use of genetically engineered mice with knockout of p53 in the brain, for example, demonstrated the significant role of apoptosis in observed volume loss immediately following CRT, though its elimination did not generally rescue smaller long-term volumes. ${ }^{63}$ Other functional and microstructural neuroimaging approaches, including rsfMRI and DTI as visualized in Figure 4, are also applicable.

As in clinical studies, animal neuroimaging results can be associated with cognitive outcome measures. Using a juvenile rat model of late-term CRT-related cognitive effects, Tang et al. demonstrated smaller gray and white matter volumes on MRI, higher brain glucose uptake via PET, and lower FA and global and local structural connectome (brain network) organization as measured by DTI. Irradiated rats showed significantly lower performance on the five-choice serial reaction time task, a measure of executive function that has a human analogue (i.e., Continuous Performance Test). Performance on the five-choice task was correlated with several structural connectome organization metrics as well as DTI fiber number and FA. Hypoconnectivity was observed in frontal, commissural, and subcortical regions, while hyperconnectivity was noted in hippocampal, fornix, and perirhinal areas. ${ }^{64}$ CRT-treated rats randomized to voluntary exercise for 10 days showed improvement in global connectome organization. Interestingly, exercise did not improve regional connections damaged by CRT, but instead appeared to alter connectivity between surrounding regions. ${ }^{65}$

Chemotherapy also alters brain structure and function in animal models. Since chemotherapy is typically delivered as a combination of several agents, separation into its component parts in animal models, along with comparison to human neuroimaging results, may help isolate how individual drug components contribute to altered brain development. ${ }^{66}$ One interesting example is cisplatin, which is used to treat several pediatric cancers. Cisplatintreated mice demonstrated deficits during puzzle box, novel object/place recognition, and Ymaze tests compared to saline-treated mice. Cisplatin-treated mice also showed altered functional connectome organization as measured by rsfMRI obtained under isoflurane 
anesthesia: hypoconnectivity was seen in frontal, striatal, and orbital regions; hyperconnectivity was demonstrated in hippocampal, entorhinal, cerebellar, and cingulate areas. Treatment with nasally administered mesenchymal stem cells improved cognitive performance and brain function. ${ }^{67}$

\section{Discussion}

Neurobiologic changes associated with pediatric cancer involve both increases and decreases in indices of brain structure and function. These effects tend to be diffuse, affecting gray matter structure and associated functional activation and connectivity of cortical and subcortical areas, as well as large WM pathways. Smaller volume in frontal, parietal, and temporal regions is a common finding across studies, which seems to correspond to poorer functional performance in other studies. Functional MRI studies have identified alterations both prior to and following systemic treatments, linked to clinical factors. However, methodological and treatment variations make direct comparisons between these studies difficult.

Brain structure and function are known to be related. If neurons (gray matter) are injured, specialized information processing (function) may be disrupted, while WM (axon) injury can result in loss of communication between specialized neuronal communities. Further, structure helps constrain the dynamic nature of functional networks to maintain the critical balance between stability and flexibility that supports core processes but also allows new learning. ${ }^{68}$ To date, few studies of pediatric cancer have included evaluation of both structure and function together, which could provide a more complete picture of the neural mechanisms underlying cognitive impairment. This may be especially important for young childhood cancer patients, where ongoing brain development is likely to result in different brain toxicities and repair potential than are observed in adults.

The interpretation of brain imaging metrics is highly context dependent. For example, studies have reported increased functional activation in areas that are observed to have reduced volume or activation in other studies. Variability in functional activation can reflect differences in underlying image contrast (possibly linked to blood flow), in the nature of the task (if present), or in information processing or use/efficiency of neural resources, depending 
on the circumstances. Moreover, the association between neuroimaging metrics and behavior may be nonlinear, ${ }^{45}$ so that compromised or compensatory changes may both underlie cognitive deficits. Regional differences in neuroimaging results add to the complexity of interpretation. It will be increasingly important to study the overall pattern of structural/functional changes, including modifiable factors that contribute to this pattern.

Hyperconnectivity was noted in multiple studies, both clinical and preclinical. The significance of this finding needs further investigation. The increased connectivity may be a means of providing additional resources for supporting neural activity after loss of connections, consistent with findings of elevated glucose uptake. This might provide compensation for easier tasks but fail during harder challenges. On the other hand, increased connectivity may indicate a more randomly organized, "noisy" brain network, ${ }^{69}$ possibly the result of altered or arrested neurodevelopment and impairment of normal synaptic pruning. This may manifest in inconsistent use of cognitive strategies, emotional dysregulation, distractibility, perseveration and/or cognitive inflexibility. Further study to elucidate the implications of hyperconnectivity will be important to understanding brain development after childhood cancer.

Currently, there are no standardized interventions for cancer-related cognitive impairment. Neuroimaging studies to date are largely cross-sectional and focused on a limited number of measures in late childhood or adulthood. Though compliance during neuroimaging sessions is a concern in younger patients, longitudinal neuroimaging before, during and early after treatment, where feasible, will provide a better understanding of the etiology of cognitive impairment. Multimodal neuroimaging, particularly in conjunction with human and animal transcriptome studies, will further aid in determining relationships among observed brain changes and the underlying mechanisms. Finally, to address individual risk factors and account for confounds, studies with larger samples will be required to be representative of survivors of various childhood cancer types. With this basis, neuroimaging could provide a much-needed early marker of effectiveness - or even guide optimal dosing - for multi-site clinical trials assaying interventions designed to prevent or treat cancer-related cognitive impairment.

\section{Acknowledgements}


SRK acknowledges support from National Institutes of Health: National Cancer Institute grants R01CA226080 and R01CA172145. CS acknowledges support from the Kinderkankerfonds Leuven. BCM acknowledges support from the National Institutes of Health: National Cancer Institute grants R35CA197289, R01CA129769, and R01CA244673 and National Institute on Aging grants P30AG010133 and R01AG019771. BJN acknowledges support from the Ontario Institute for Cancer Research through funding provided by the Government of Ontario (IA-024) and by the Canadian Institutes of Health Research (156250, 158622, 168925). 


\section{Figure Captions}

Figure 1: Neuroimaging findings in childhood cancer survivors. Both childhood cancer and its treatment have the potential to introduce toxicities in the brain. Neuroimaging in survivorship represents the net effect of these cancer- and treatment-related injuries, as well as the brain's intrinsic repair processes in the context of ongoing development. Key findings highlighted in the text are depicted under the macrostructure, microstructure, neuronal activity, and vasculature and metabolism headings. Up ( $\uparrow)$ and down $(\downarrow)$ arrows indicate reported increases or decreases in the corresponding metric. Where both arrows are indicated $(\downarrow \uparrow)$, both decreases and increases have been reported, either due to regional variations or due to different literature reports. The dash (-) indicates reports of no change in outcome. Regional alterations measured by neuroimaging have broader implications for the whole brain network, which may be assessed through connectome measures, and for cognition and behavior, and can significantly impact quality of life in childhood cancer survivors. Abbreviations: sMRI: structural MRI; GM: gray matter; WM: white matter; ctx thk: cortical thickness; ctx area: cortical area; dMRI: diffusion MRI; FA: fractional anisotropy; RD: radial diffusivity; MD: mean diffusivity; MK: mean kurtosis; ODI: orientation dispersion index; AFD: apparent fiber density; MTR: magnetization transfer ratio; MWF: myelin water fraction; MEG: magnetoencephalography; osc freq: oscillatory frequency; fMRI: functional MRI; rs conn: resting-state connectivity; CBF: cerebral blood flow; PET: positron emission tomography; FDG: fluorodeoxyglucose.

Figure 2: Anatomical MRI and image processing for assessment of brain structure in childhood cancer survivors. Based on anatomical scans, typically $\mathrm{T}_{1}$-weighted or a combination of $\mathrm{T}_{1}$ - and $\mathrm{T}_{2}$-weighted MRI scans, morphometric analyses can be performed to assess global and regional changes in brain structure, including volumes, areas, thicknesses, etc. In the middle, voxelbased morphometry is depicted, in which the anatomical images are processed to produce tissue type images in which the intensity of a voxel represents the relative proportion of each tissue type in that voxel. These can be statistically compared between groups (e.g., childhood cancer survivor versus control). At right, surface-based morphometry is illustrated for quantification of the cortex, including characterization of its folding. Measures include cortical thickness, gyrification, and sulcus depth.

Figure 3: Diffusion MRI and modeling to estimate WM microstructure changes in childhood cancer survivors. (A) Diffusion of water in WM is affected by myelin and axons. Modeling of this effect allows parameter maps to be generated which are sensitive to different microstructural changes. Imaging parameter maps sensitive to white matter microstructural alterations in childhood cancer survivors and discussed in this review are depicted (FA: Fractional Anisotropy; 
MD: Mean Diffusivity; RD: Radial Diffusivity; MK: Mean Kurtosis; ODI: Orientation Dispersion Index; AFD: Apparent Fiber Density). At right, alternative MRI contrasts (MWF: Myelin Water Fraction; MT: magnetization transfer imaging) known to be sensitive to myelin content are also depicted. (B) Advanced diffusion weighted imaging techniques allow characterization of WM architecture using the diffusion of water molecules, which is highly directional (anisotropic) in healthy WM (i) because of axonal membranes and myelin sheaths. Axonal loss (ii) or demyelination (iii) results in less anisotropic diffusion and therefore changes in diffusion imaging parameter maps and related structural brain connectivity. Sample results are shown at bottom and include: (iv) brain regions showing significantly decreased FA in ALL survivors who received CSI compared to healthy controls ${ }^{25}$ (reprinted with permission from Journal of Clinical Oncology); (v) decreased AFD in the corpus callosum in childhood sarcoma survivors who received systemic chemotherapy compared to healthy controls ${ }^{43}$ (reprinted with permission from Human Brain Mapping); and (vi) structural connectome organization change in young survivors of ALL who received CNS-directed chemotherapy ${ }^{45}$ (reprinted with permission from Brain Connectivity).

Figure 4: Sample preclinical neuroimaging results in models of childhood cancer survivorship. At left, a murine functional connectome derived from resting state $\mathrm{fMRI}$ is depicted. The connectome models the brain as a network of nodes (regions) with joining edges (connections). Relative size of the spheres (i.e., nodes) indicates the number of edges (gray lines) passing through them. At right, a color fiber map is used for visualization of murine DTI data, in which colors indicate virtual white matter streamline direction (red: right-left; blue: inferior-superior; green: anterior-posterior). 


\section{References}

1. Sleurs C, Deprez S, Emsell L, et al: Chemotherapy-induced neurotoxicity in pediatric solid nonCNS tumor patients: An update on current state of research and recommended future directions. Crit Rev Oncol Hematol 103:37-48, 2016

2. Zhang $X$, Braun $U$, Tost $H$, et al: Data-Driven Approaches to Neuroimaging Analysis to Enhance Psychiatric Diagnosis and Therapy. Biol Psychiatry Cogn Neurosci Neuroimaging , 2020

3. Fox MD, Buckner RL, Liu H, et al: Resting-state networks link invasive and noninvasive brain stimulation across diverse psychiatric and neurological diseases. Proc Natl Acad Sci U S A 111:E4367-75, 2014

4. Gabrieli JDE, Ghosh SS, Whitfield-Gabrieli S: Prediction as a humanitarian and pragmatic contribution from human cognitive neuroscience. Neuron 85:11-26, 2015

5. Partap S, Russo S, Esfahani B, et al: A Review of Chronic Leukoencephalopathy among Survivors of Childhood Cancer. Pediatr Neurol 101:2-10, 2019

6. Cheung YT, Sabin ND, Reddick WE, et al: Leukoencephalopathy and long-term neurobehavioural, neurocognitive, and brain imaging outcomes in survivors of childhood acute lymphoblastic leukaemia treated with chemotherapy: a longitudinal analysis. Lancet Haematol 3:e456-e466, 2016

7. Reulen RC, Guha J, Bright CJ, et al: Risk of cerebrovascular disease among 13,457 five-year survivors of childhood cancer: a population based cohort study [Internet]. Int J Cancer , 2020 
8. Kähkönen M, Harila-Saari A, Metsähonkala L, et al: Cerebral blood flow and glucose metabolism in long-term survivors of childhood acute lymphoblastic leukaemia. Eur J Cancer 35:1102-1108, 1999

9. Mulhern RK, Palmer SL, Reddick WE, et al: Risks of young age for selected neurocognitive deficits in medulloblastoma are associated with white matter loss. J Clin Oncol 19:472-479, 2001

10. Reddick WE, White HA, Glass JO, et al: Developmental model relating white matter volume to neurocognitive deficits in pediatric brain tumor survivors. Cancer 97:2512-2519, 2003

11. Nagel BJ, Palmer SL, Reddick WE, et al: Abnormal hippocampal development in children with medulloblastoma treated with risk-adapted irradiation. AJNR Am J Neuroradiol 25:15751582,2004

12. Liu AK, Marcus KJ, Fischl B, et al: Changes in Cerebral Cortex of Children Treated for Medulloblastoma. International Journal of Radiation Oncology*Biology*Physics 68:992-998, 2007

13. Nieman BJ, de Guzman AE, Gazdzinski LM, et al: White and Gray Matter Abnormalities After Cranial Radiation in Children and Mice. Int J Radiat Oncol Biol Phys 93:882-891, 2015

14. Armstrong GT, Reddick WE, Petersen RC, et al: Evaluation of memory impairment in aging adult survivors of childhood acute lymphoblastic leukemia treated with cranial radiotherapy. J Natl Cancer Inst 105:899-907, 2013 
15. Zhou C, Zhuang $Y$, Lin $X$, et al: Changes in neurocognitive function and central nervous system structure in childhood acute lymphoblastic leukaemia survivors after treatment: a metaanalysis. Br J Haematol 188:945-961, 2020

16. Reddick WE, Taghipour DJ, Glass JO, et al: Prognostic factors that increase the risk for reduced white matter volumes and deficits in attention and learning for survivors of childhood cancers. Pediatr Blood Cancer 61:1074-1079, 2014

17. Zeller B, Tamnes CK, Kanellopoulos A, et al: Reduced neuroanatomic volumes in long-term survivors of childhood acute lymphoblastic leukemia. J Clin Oncol 31:2078-2085, 2013

18. van der Plas E, Schachar RJ, Hitzler J, et al: Brain structure, working memory and response inhibition in childhood leukemia survivors. Brain Behav 7:e00621, 2016

19. Reddick WE, Shan ZY, Glass JO, et al: Smaller white-matter volumes are associated with larger deficits in attention and learning among long-term survivors of acute lymphoblastic leukemia. Cancer 106:941-949, 2006

20. Kesler SR, Tanaka H, Koovakkattu D: Cognitive reserve and brain volumes in pediatric acute Iymphoblastic leukemia. Brain Imaging Behav 4:256-269, 2010

21. Tamnes CK, Zeller B, Amlien IK, et al: Cortical surface area and thickness in adult survivors of pediatric acute lymphoblastic leukemia. Pediatr Blood Cancer 62:1027-1034, 2015

22. Krull KR, Cheung YT, Liu W, et al: Chemotherapy Pharmacodynamics and Neuroimaging and Neurocognitive Outcomes in Long-Term Survivors of Childhood Acute Lymphoblastic Leukemia. 
J Clin Oncol 34:2644-2653, 2016

23. Phillips NS, Cheung YT, Glass JO, et al: Neuroanatomical abnormalities related to dexamethasone exposure in survivors of childhood acute lymphoblastic leukemia. Pediatr Blood Cancer 67:e27968, 2020

24. Sleurs C, Blommaert J, Batalle D, et al: Cortical thinning and altered functional brain coherence in survivors of childhood sarcoma. Brain Imaging Behav In Press, 2020

25. Schuitema I, Deprez S, Van Hecke W, et al: Accelerated aging, decreased white matter integrity, and associated neuropsychological dysfunction 25 years after pediatric lymphoid malignancies. J Clin Oncol 31:3378-3388, 2013

26. Follin C, Svärd D, van Westen D, et al: Microstructural white matter alterations associated to neurocognitive deficits in childhood leukemia survivors treated with cranial radiotherapy - a diffusional kurtosis study. Acta Oncol 58:1021-1028, 2019

27. Mabbott DJ, Noseworthy MD, Bouffet $E$, et al: Diffusion tensor imaging of white matter after cranial radiation in children for medulloblastoma: correlation with IQ. Neuro Oncol 8:244252,2006

28. Palmer SL, Glass JO, Li Y, et al: White matter integrity is associated with cognitive processing in patients treated for a posterior fossa brain tumor. Neuro Oncol 14:1185-1193, 2012

29. Khong P-LL, Leung LHT, Fung ASM, et al: White matter anisotropy in post-treatment childhood cancer survivors: preliminary evidence of association with neurocognitive function. J 
Clin Oncol 24:884-890, 2006

30. Redmond KJ, Hildreth M, Sair HI, et al: Association of Neuronal Injury in the Genu and Body of Corpus Callosum After Cranial Irradiation in Children With Impaired Cognitive Control: A Prospective Study. Int J Radiat Oncol Biol Phys 101:1234-1242, 2018

31. Makola M, Douglas Ris M, Mahone EM, et al: Long-term effects of radiation therapy on white matter of the corpus callosum: a diffusion tensor imaging study in children. Pediatr Radiol 47:1809-1816, 2017

32. Darling SJ, De Luca C, Anderson V, et al: White Matter Microstructure and Information Processing at the Completion of Chemotherapy-Only Treatment for Pediatric Acute Lymphoblastic Leukemia. Dev Neuropsychol 43:385-402, 2018

33. Sabin ND, Cheung YT, Reddick WE, et al: The Impact of Persistent Leukoencephalopathy on Brain White Matter Microstructure in Long-Term Survivors of Acute Lymphoblastic Leukemia Treated with Chemotherapy Only. AJNR Am J Neuroradiol 39:1919-1925, 2018

34. ElAlfy M, Ragab I, Azab I, et al: Neurocognitive outcome and white matter anisotropy in childhood acute lymphoblastic leukemia survivors treated with different protocols. Pediatr Hematol Oncol 31:194-204, 2014

35. Aukema EJ, Caan MWA, Oudhuis N, et al: White matter fractional anisotropy correlates with speed of processing and motor speed in young childhood cancer survivors. Int J Radiat Oncol Biol Phys 74:837-843, 2009 
36. Liu F, Scantlebury N, Tabori U, et al: White matter compromise predicts poor intellectual outcome in survivors of pediatric low-grade glioma. Neuro Oncol 17:604-613, 2015

37. Peterson RK, Tabori U, Bouffet E, et al: Predictors of neuropsychological late effects and white matter correlates in children treated for a brain tumor without radiation therapy. Pediatr Blood Cancer 66:e27924, 2019

38. Banerjee P, Rossi MG, Anghelescu DL, et al: Association Between Anesthesia Exposure and Neurocognitive and Neuroimaging Outcomes in Long-term Survivors of Childhood Acute Lymphoblastic Leukemia. JAMA Oncol In Press, 2019

39. Billiet T, Elens I, Sleurs C, et al: Brain Connectivity and Cognitive Flexibility in Nonirradiated Adult Survivors of Childhood Leukemia. J Natl Cancer Inst 110:905-913, 2018

40. Brinkman TM, Reddick WE, Luxton J, et al: Cerebral white matter integrity and executive function in adult survivors of childhood medulloblastoma. Neuro Oncol 14 Suppl 4:iv25-36, 2012

41. Glass JO, Ogg RJ, Hyun JW, et al: Disrupted development and integrity of frontal white matter in patients treated for pediatric medulloblastoma. Neuro Oncol 19:1408-1418, 2017

42. Partanen $M$, Bouffet $E$, Laughlin $S$, et al: Early changes in white matter predict intellectual outcome in children treated for posterior fossa tumors. Neuroimage Clin 20:697-704, 2018

43. Sleurs C, Lemiere J, Christiaens D, et al: Advanced MR diffusion imaging and chemotherapyrelated changes in cerebral white matter microstructure of survivors of childhood bone and soft 
tissue sarcoma? Hum Brain Mapp 39:3375-3387, 2018

44. Na S, Li L, Crosson B, et al: White matter network topology relates to cognitive flexibility and cumulative neurological risk in adult survivors of pediatric brain tumors. Neuroimage Clin $20: 485-497,2018$

45. Kesler SR, Gugel M, Huston-Warren E, et al: Atypical Structural Connectome Organization and Cognitive Impairment in Young Survivors of Acute Lymphoblastic Leukemia. Brain Connect 6:273-282, 2016

46. Yamamoto A, Miki Y, Adachi S, et al: Whole brain magnetization transfer histogram analysis of pediatric acute lymphoblastic leukemia patients receiving intrathecal methotrexate therapy. Eur J Radiol 57:423-427, 2006

47. Shrot S, Abebe-Campino G, Toren A, et al: Fluorodeoxyglucose Detected Changes in Brain Metabolism After Chemotherapy in Pediatric Non-Hodgkin Lymphoma. Pediatr Neurol 92:3742,2019

48. Tauty A, Noblet V, Paillard C, et al: Evaluation of the effects of chemotherapy on brain glucose metabolism in children with Hodgkin's lymphoma. Ann Nucl Med 33:564-569, 2019

49. Daams M, Schuitema I, van Dijk BW, et al: Long-term effects of cranial irradiation and intrathecal chemotherapy in treatment of childhood leukemia: a MEG study of power spectrum and correlated cognitive dysfunction. BMC Neurol 12:84, 2012

50. Robinson KE, Pearson MM, Cannistraci CJ, et al: Neuroimaging of executive function in 
survivors of pediatric brain tumors and healthy controls. Neuropsychology 28:791-800, 2014

51. King TZ, Na S, Mao H: Neural Underpinnings of Working Memory in Adult Survivors of Childhood Brain Tumors. J Int Neuropsychol Soc 21:494-505, 2015

52. Conklin HM, Ness KK, Ashford JM, et al: Cognitive Performance, Aerobic Fitness, Motor Proficiency, and Brain Function Among Children Newly Diagnosed With Craniopharyngioma. J Int Neuropsychol Soc 25:413-425, 2019

53. Fellah S, Cheung YT, Scoggins MA, et al: Brain Activity Associated With Attention Deficits Following Chemotherapy for Childhood Acute Lymphoblastic Leukemia. J Natl Cancer Inst 111:201-209, 2018

54. Hu Z, Zou D, Mai H, et al: Altered brain function in new onset childhood acute lymphoblastic leukemia before chemotherapy: A resting-state fMRI study. Brain Dev 39:743-750, 2017

55. Chen $\mathrm{H}$, Wang $\mathrm{L}$, King TZ, et al: Increased frontal functional networks in adult survivors of childhood brain tumors. Neuroimage Clin 11:339-346, 2016

56. Kesler SR, Ogg R, Reddick WE, et al: Brain Network Connectivity and Executive Function in Long-Term Survivors of Childhood Acute Lymphoblastic Leukemia. Brain Connect 8:333-342, 2018

57. Zou $\mathrm{P}$, Conklin HM, Scoggins MA, et al: Functional MRI in medulloblastoma survivors supports prophylactic reading intervention during tumor treatment. Brain Imaging Behav $10: 258-271,2016$ 
58. Cox E, Bells S, Timmons BW, et al: A controlled clinical crossover trial of exercise training to improve cognition and neural communication in pediatric brain tumor survivors. Clin Neurophysiol 131:1533-1547, 2020

59. Zou P, Li Y, Conklin HM, et al: Evidence of change in brain activity among childhood cancer survivors participating in a cognitive remediation program. Arch Clin Neuropsychol 27:915-929, 2012

60. Conklin HM, Ogg RJ, Ashford JM, et al: Computerized Cognitive Training for Amelioration of Cognitive Late Effects Among Childhood Cancer Survivors: A Randomized Controlled Trial. J Clin Oncol 33:3894-3902, 2015

61. de Guzman AE, Gazdzinski LM, Alsop RJ, et al: Treatment age, dose and sex determine neuroanatomical outcome in irradiated juvenile mice. Radiat Res 183:541-549, 2015

62. Perez EC, Rodgers SP, Inoue T, et al: Olfactory Memory Impairment Differs by Sex in a Rodent Model of Pediatric Radiotherapy. Front Behav Neurosci 12:158, 2018

63. de Guzman AE, Ahmed M, Li Y-Q, et al: p53 Loss Mitigates Early Volume Deficits in the Brains of Irradiated Young Mice. Int J Radiat Oncol Biol Phys 103:511-520, 2019

64. Tang TT, Zawaski JA, Kesler SR, et al: A comprehensive preclinical assessment of late-term imaging markers of radiation-induced brain injury. Neurooncol Adv 1:vdz012, 2019

65. Sahnoune I, Inoue T, Kesler SR, et al: Exercise ameliorates neurocognitive impairments in a translational model of pediatric radiotherapy. Neuro Oncol 20:695-704, 2018 
66. Spencer Noakes TL, Przybycien TS, Forwell A, et al: Brain Development and Heart Function after Systemic Single-Agent Chemotherapy in a Mouse Model of Childhood Leukemia Treatment. Clin Cancer Res 24:6040-6052, 2018

67. Chiu GS, Boukelmoune N, Chiang ACA, et al: Nasal administration of mesenchymal stem cells restores cisplatin-induced cognitive impairment and brain damage in mice. Oncotarget $9: 35581-35597,2018$

68. Telesford QK, Lynall M-E, Vettel J, et al: Detection of functional brain network reconfiguration during task-driven cognitive states. Neuroimage 142:198-210, 2016

69. Bassett DS, Bullmore ET: Small-World Brain Networks Revisited. Neuroscientist 23:499-516, 2017 